\title{
Fabrication and in vitro/in vivo evaluation of amorphous andrographolide nanosuspensions stabilized by $D$ - $\alpha$-tocopheryl polyethylene glycol 1000 succinate/sodium lauryl sulfate
}

\author{
This article was published in the following Dove Press journal: \\ International Journal of Nanomedicine \\ 7 February 2017 \\ Number of times this article has been viewed
}

\author{
Hongzhi Qiao,2,* \\ Lihua Chen ${ }^{3, *}$ \\ Tianqi Rui ${ }^{1,2}$ \\ Jingxian Wang ${ }^{1,2}$ \\ Ting Chen ${ }^{1,2}$ \\ Tingming $\mathrm{Fu}^{1,2}$ \\ Junsong $\mathrm{Li}^{1,2}$ \\ Liuqing $\mathrm{Di}^{1,2}$ \\ 'College of Pharmacy, Nanjing \\ University of Chinese Medicine, \\ ${ }^{2}$ Jiangsu Engineering Research Center \\ for Efficient Delivery System of TCM, \\ Nanjing, ${ }^{3}$ Key Laboratory of Modern \\ Preparation of Traditional Chinese \\ Medicine, Ministry of Education, \\ Jiangxi University of Traditional \\ Chinese Medicine, Nanchang, China \\ *These authors contributed equally \\ to this work
}

\begin{abstract}
Andrographolide (ADG) is a diterpenoid isolated from Andrographis paniculata with a wide spectrum of biological activities, including anti-inflammatory, anticancer and hepatoprotective effects. However, its poor water solubility and efflux by P-glycoprotein have resulted in lower bioavailability. In this study, ADG nanosuspensions (ADG-NS) were prepared using a wet media milling technique followed by freeze drying. D- $\alpha$-Tocopheryl polyethylene glycol 1000 succinate (TPGS), a surfactant that inhibits P-glycoprotein function, and sodium lauryl sulfate were used as surface stabilizers. A Box-Behnken design was used to optimize the nanosuspension preparation. The products of these optimal preparation conditions were amorphous and possessed much faster dissolution in vitro than a coarse powder of ADG. The particle size and redispersibility index of the freeze-dried ADG-NS were 244.6 $\pm 3.0 \mathrm{~nm}$ and $113 \% \pm 1.14 \%(n=3)$, respectively. A short-term stability study indicated that the freezedried ADG-NS could remain highly stable as nanosuspensions during the testing period. A test of transport across a Caco-2 cell monolayer revealed that the membrane permeability $\left(P_{\text {app }}\right)$ of ADG-NS was significantly higher than the permeability of the ADG coarse powder or ADG-NS without TPGS $(P<0.01)$. Compared to the ADG coarse powder, a physical mixture, commercial dripping pills and ADG-NS without TPGS, ADG-NS exhibited significantly higher plasma exposure with significant enhancements in $C_{\max }$ and area under the curve of plasma concentration versus time from zero to the last sampling time $\left(\mathrm{AUC}_{0-t}\right)(P<0.01)$. An evaluation of the anti-inflammatory effect on Carr-induced paw edema demonstrated that the ADG-NS were more effective in reducing the rate of paw swelling, producing a greater increase in the serum levels of nitric oxide (NO), Interleukin-1 (IL-1) and tumor necrosis factor- $\alpha$ (TNF- $\alpha)(P<0.01)$ and an increase in superoxide dismutase activity $(P<0.05)$ compared to the ADG coarse powder. This study indicated that nanosuspensions could act as an effective delivery device for ADG to enhance its oral bioavailability and biological efficacy.
\end{abstract}

Keywords: andrographolide, nanosuspensions, Box-Behnken design, D- $\alpha$-tocopheryl polyethylene glycol 1000 succinate, dissolution rate, oral bioavailability

\section{Introduction}

Andrographolide (ADG) is the main active ingredient in Andrographis paniculata (Burm. f.) Nees, which is commonly used in Chinese medicine to treat inflammation. ${ }^{1-3}$ ADG can relieve fever, detoxify, diminish inflammation, stop pain and have a curative effect on bacterial and viral upper respiratory tract infections and diarrhea, which makes it honored as a natural antibiotic. ${ }^{4}$ It can also inhibit malignant tumor growth in various
College of Pharmacy, Nanjing University of Chinese Medicine, I 38 Xianlin Avenue, Nanjing 210023, China

Tel/fax $+8625858 I \quad 1517$

Email lijunsong1964@163.com 
cell lines, including breast, hepatic, cervical, colon and gastric cancers and glioblastoma in preclinical studies. ${ }^{5,6}$ The potential use of ADG has attracted wide attention in recent years. Unfortunately, the clinical use of ADG remains challenging due to its extremely poor solubility $\left(1.32 \times 10^{-7} \mathrm{M}\right.$ in water at $25^{\circ} \mathrm{C}$ ) and efflux by P-glycoprotein (P-gp), ${ }^{7-9}$ which results in low bioavailability after oral administration.

At present, there are several methods used to improve the oral absorption of ADG, including dripping pills, dispersible tablets, cyclodextrin inclusion complexes, nanoparticles and microcrystals. ${ }^{10-14}$ Although the bioavailability of ADG has been improved to some extent, its clinical use is still greatly restricted because of limited improvements in bioavailability, a low drug-loading/entrapment rate, poor stability or complicated and expensive manufacturing processes.

Nanosuspensions are crystalline nanoparticles ranging from 200 to $500 \mathrm{~nm}$ in size that are stabilized by surface stabilizers. ${ }^{15}$ They can increase the saturation solubility of a drug, the in vitro dissolution rate and likely mucoadhesion, and they can then improve the oral bioavailability. ${ }^{16,17}$ In addition, nanosuspensions have the advantages of high drug loading, the avoidance of organic solvents, enhanced stability and low toxicity in comparison to other DDS, such as liposomes, polymer nanoparticles, lipid nanoparticles and formulations with cosolvents. ${ }^{18,19}$ Since the 1990 s, many commercial nanosuspension products, including Rapamune ${ }^{\circledR}$, Emend $^{\circledR}$, Tricor $^{\circledR}$, Megace ES ${ }^{\circledR}$, Avinza $^{\circledR}$, Focalin XR $^{\circledR}$, Ritalin ${ }^{\circledR}$ and Zanaflex Capsules ${ }^{\mathrm{TM}}$, have been marketed successfully. ${ }^{20}$

Due to the changing Gibbs free energy, thermodynamically unstable nanosuspensions would be inclined toward agglomeration or crystal growth due to Ostwald ripening. The proper selection of stabilizers is required. The most common approaches for stabilization are steric and/or electrostatic techniques, eg, placing ionic surfactants and/or charged polymers onto the particle surface. ${ }^{21}$

Recently, many studies have proven that D- $\alpha$-tocopheryl polyethylene glycol 1000 succinate (TPGS), a nonionic surfactant commonly used in pharmaceutical formulations, can disrupt the function of P-gp. ${ }^{22,23}$ Because this surfactant reduces surface energy and inhibits $\mathrm{P}$-gp function, the use of TPGS coupled with sodium lauryl sulfate (SLS) as a stabilizer in nanosuspension systems could achieve stabilization by means of their steric and electrostatic properties as well as higher absorption across the intestinal epithelium by means of inhibiting the P-gp efflux function.

Quality by design (specifically, design of experiment [DoE]) is a versatile approach to understanding the effect of critical process parameters and to optimizing the process conditions. ${ }^{24,25}$ DoE helps to identify critical and noncritical parameters affecting product quality. Furthermore, the results of DoE afford the quantification of any interactions between critical parameters based on the responses. DoE can also be utilized to predict the desired quality attributes within the design space. ${ }^{26}$

In this study, ADG nanosuspensions (ADG-NS) were prepared by wet media milling using TPGS coupled with SLS as a stabilizer. The formulation and processing of ADG-NS were optimized by Box-Behnken design (BBD), and the suspensions were then systematically characterized and evaluated in vitro and in vivo. Finally, the anti-inflammatory effects of ADG-NS on Carr-induced paw edema were also investigated.

\section{Materials and methods Materials}

ADG and bilobalide were purchased from Shanghai Yuanye Bio-Technology Co., Ltd. (Shanghai, China) and had a mass purity of $>98.0 \%$. TPGS was provided by Sigma-Aldrich (St Louis, MO, USA). SLS was obtained from Aladdin Industrial Co., Ltd. (Shanghai, China). ADG dripping pills were purchased from Tasly Pharmaceutical Group Co., Ltd. (Tianjin, China). Aspirin was purchased from Bayer Healthcare Co., Ltd. (Shanghai, China). Carrageen was obtained from Sigma-Aldrich (St. Louis, MO, USA). The diagnostic kits for superoxide dismutase (SOD) activities and for nitric oxide (NO), Interleukin-1 (IL-1), and tumor necrosis factor- $\alpha$ (TNF- $\alpha$ ) levels were purchased from Nanjing Jiancheng Bioengineering Research Institute (Nanjing, China). The Caco-2 cell line was obtained from the American Type Culture Collection (ATCC, Manassas, VA, USA). Dulbecco's Modified Eagle's Medium (DMEM), nonessential amino acids and fetal bovine serum (FBS) were supplied by Gibco (Grand Island, NY, USA). Purified water was prepared using a Milli- $Q^{\circledR}$ purification system (Millipore, Billerica, MA, USA). Other chemical reagents of analytical grade or better were obtained from Sinopharm Chemical Reagent Co., Ltd. (Nanjing, China).

\section{Methods}

\section{Preparation of ADG-NS}

The ADG-NS were prepared using a wet media milling approach. One gram of ADG coarse powder was dispersed in $200 \mathrm{~mL}$ of an aqueous solution containing TPGS and SLS at specific concentrations as designed by the DoE under magnetic stirring. The obtained mixture was formed into microparticles using a high shear homogenizer (BRT, B25, Germany) at 16,000 rpm for $5 \mathrm{~min}$. The resulting microsuspensions were then wet-milled with zirconium oxide 
beads (0.3-0.4 mm in diameter) using an NT-0.3L Mill (Dongguan Longly Machinery Factory, Dongguan, China) to obtain liquid ADG-NS. The agitator speed and milling time were designed by the DoE. The processing temperature was maintained $<25^{\circ} \mathrm{C}$ by passing cooled water through the outer jacket throughout the process.

To enhance the chemical and physical stabilities, liquid ADG-NS were lyophilized before storage and subsequent use. Briefly, the ADG-NS, with $1 \%(\mathrm{w} / \mathrm{v})$ mannitol as a cryoprotectant, were rapidly cooled, kept at $-80^{\circ} \mathrm{C}$ for $12 \mathrm{~h}$, then transferred to a LABCONCO 6-L freeze-dryer (Labconco Corporation, Kansas City, KS, USA) and dried at $-30,-10$, 0 and $25^{\circ} \mathrm{C}$ for $10,5,2$ and $2 \mathrm{~h}$, respectively, at 1 psi pressure to obtain a dried ADG-NS powder.

\section{Experimental design}

Initial screening studies were carried out to evaluate the effect of processing parameters and formulation parameters on the particle size and polydispersity index (PDI) of the resulting ADG-NS. The TPGS and SLS concentrations were identified as critical formulation parameters, while the agitator speed and milling time were critical process parameters. The DoE was used systematically to evaluate and optimize the selected process and formulation parameters at three levels $(-1,0$ and +1 ). Based on the number of factors and their levels, BBD was selected to investigate their effects on the critical quality attributes (CQAs) of the nanosuspensions. The particle size and PDI were chosen to be dependent factors. Independent factors and the levels used in this study are shown in Table 1. The design contains 29 experimental runs; ie, 24 factorial points and five center points were generated, as shown in Table 2, and analyzed by Design-Expert ${ }^{\circledR}$ V8.0.6 (Stat-Ease Inc., Minneapolis, MN, USA).

Table I Independent factors and their coded levels of BBD

\begin{tabular}{|c|c|c|c|}
\hline \multicolumn{2}{|l|}{ Independent factors } & \multicolumn{2}{|l|}{ Design level } \\
\hline Actual parameters & Coded & Actual value & Coded level \\
\hline \multirow{3}{*}{ TPGS concentration $(\%, w / v)$} & $A$ & 0.1 & -1 \\
\hline & & 0.25 & 0 \\
\hline & & 0.4 & 1 \\
\hline \multirow[t]{3}{*}{ SLS concentration $(\%, w / v)$} & B & 0.05 & -1 \\
\hline & & 0.2 & 0 \\
\hline & & 0.35 & 1 \\
\hline \multirow[t]{3}{*}{ Agitator speed (rpm) } & $C$ & 1,000 & -1 \\
\hline & & 1,500 & 0 \\
\hline & & 2,000 & $\mathrm{I}$ \\
\hline \multirow[t]{3}{*}{ Milling time (min) } & $D$ & 20 & -1 \\
\hline & & 60 & 0 \\
\hline & & 100 & 1 \\
\hline
\end{tabular}

Abbreviations: BBD, Box-Behnken design; SLS, sodium lauryl sulfate; TPGS, D- $\alpha$ tocopheryl polyethylene glycol 1000 succinate.
The mean particle size and PDI of ADG-NS were determined by photon correlation spectroscopy (PCS) (Zetasizer Nano ZS; Malvern Instruments, Malvern, UK) at room temperature. Each sample was measured three times. The surface charge of the particles was assessed by measuring the zeta potential using the same instrument at $25^{\circ} \mathrm{C}$ by applying the fields of $20 \mathrm{~V} / \mathrm{cm}$.

\section{Characterization of optimized ADG-NS \\ Reconstitution test}

A $5 \mathrm{~g}$ sample of the freeze-dried ADG-NS powder was dispersed in a $250 \mathrm{~mL}$ dissolution cup with $100 \mathrm{~mL}$ of purified water and stirred with a paddle at $100 \mathrm{rpm}$ for $5 \mathrm{~min}$. The mean particle size and PDI of ADG-NS were determined as described earlier.

The redispersibility index (RDI) was used to evaluate the redispersibility of the freeze-dried ADG-NS powders and is calculated as follows:

$$
\mathrm{RDI}=\frac{\mathrm{D}}{\mathrm{D}_{0}} \times 100 \%
$$

where $\mathrm{D}$ represents the mean particle size of the redispersed freeze-dried ADG-NS powder and $\mathrm{D}_{0}$ is the particle size of the liquid ADG-NS prior to freeze drying. When the RDI value is $\sim 100 \%$, the freeze-dried ADG-NS powder is considered to be completely redispersed..$^{27}$

\section{Scanning electron microscopy (SEM)}

The morphology of the ADG coarse powder and the freezedried ADG-NS was evaluated using SEM (JSM-5610LV; Rigaku, Tokyo, Japan). Prior to examination, the samples were mounted onto metal stubs using double-sided adhesive tape and sputtered with a thin layer of gold under vacuum. The SEM was operated at an acceleration voltage of $10 \mathrm{kV}$.

\section{Differential scanning calorimetry (DSC)}

DSC measurements were made using a NETZSCH DSC-204 differential scanning calorimeter (NETZSCH, Selb, Germany). The samples, including the ADG coarse powder, TPGS, SLS, a physical mixture of the ADG coarse powder, TPGS and SLS (PM) and the freeze-dried ADG-NS, were placed in a standard aluminum crucible fitted with a perforated lid for scanning. A heating rate of $10^{\circ} \mathrm{C} / \mathrm{min}$ was used in the range of $30^{\circ} \mathrm{C}-300^{\circ} \mathrm{C}$, and an empty pan was used as a reference.

\section{Powder X-ray diffraction (PXRD)}

The PXRD patterns of ADG coarse powder, TPGS, SLS, PM and the freeze-dried ADG-NS were analyzed by a powder $\mathrm{X}$-ray diffractometer (D/Max-2500PC; Rigaku) with a $\mathrm{Cu}$ 
Table 2 Effect of selected process and formulation parameters on particle size distribution of ADG-NS

\begin{tabular}{|c|c|c|c|c|c|c|}
\hline \multirow[t]{2}{*}{ Run } & \multicolumn{4}{|l|}{ Critical factors } & \multicolumn{2}{|l|}{ Responses } \\
\hline & $\begin{array}{l}\text { TPGS concentration } \\
(\%, w / v)\end{array}$ & $\begin{array}{l}\text { SLS concentration } \\
(\%, w / v)\end{array}$ & $\begin{array}{l}\text { Agitator } \\
\text { speed (rpm) }\end{array}$ & $\begin{array}{l}\text { Milling } \\
\text { time (min) }\end{array}$ & $\begin{array}{l}\text { Particle } \\
\text { size }(\mathrm{nm})\end{array}$ & PDI \\
\hline 1 & 0.4 & 0.2 & 2,000 & 60 & 230.4 & 0.172 \\
\hline 2 & 0.4 & 0.2 & $\mathrm{I}, 500$ & 20 & 331.6 & 0.242 \\
\hline 3 & 0.4 & 0.2 & $\mathrm{I}, 500$ & 100 & 235.1 & 0.183 \\
\hline 4 & 0.1 & 0.2 & 2,000 & 60 & 229 & 0.183 \\
\hline 5 & 0.4 & 0.05 & $\mathrm{I}, 500$ & 60 & $4 \mid 8.1$ & 0.362 \\
\hline 6 & 0.25 & 0.2 & 1,000 & 20 & 341.1 & 0.172 \\
\hline 7 & 0.25 & 0.35 & $\mathrm{I}, 500$ & 20 & 270.8 & 0.135 \\
\hline 8 & 0.25 & 0.2 & 2,000 & 20 & 290.2 & 0.252 \\
\hline 9 & 0.25 & 0.2 & $\mathrm{I}, 500$ & 60 & 228.8 & 0.114 \\
\hline 10 & 0.25 & 0.35 & 2,000 & 60 & 219.3 & 0.185 \\
\hline 11 & 0.25 & 0.35 & $\mathrm{I}, 500$ & 100 & 213.1 & 0.145 \\
\hline 12 & 0.25 & 0.2 & $\mathrm{I}, 500$ & 60 & 214.2 & 0.165 \\
\hline 13 & 0.25 & 0.05 & $\mathrm{I}, 500$ & 100 & 589.6 & 0.358 \\
\hline 14 & 0.25 & 0.2 & $\mathrm{I}, 500$ & 60 & 225.4 & 0.131 \\
\hline 15 & 0.1 & 0.05 & $\mathrm{I}, 500$ & 60 & 480.6 & 0.287 \\
\hline 16 & 0.1 & 0.2 & 1,000 & 60 & 233.7 & 0.181 \\
\hline 17 & 0.25 & 0.2 & 2,000 & 100 & 234.9 & 0.174 \\
\hline 18 & 0.4 & 0.35 & 1,500 & 60 & 229.5 & 0.151 \\
\hline 19 & 0.25 & 0.05 & 2,000 & 60 & 385.6 & 0.227 \\
\hline 20 & 0.1 & 0.2 & $\mathrm{I}, 500$ & 100 & 218.2 & 0.191 \\
\hline 21 & 0.25 & 0.2 & I,500 & 60 & 214.3 & 0.173 \\
\hline 22 & 0.25 & 0.2 & 1,000 & 100 & 227.6 & 0.151 \\
\hline 23 & 0.25 & 0.05 & $\mathrm{I}, 500$ & 20 & 592.8 & 0.29 \\
\hline 24 & 0.1 & 0.35 & 1,500 & 60 & 223.2 & 0.185 \\
\hline 25 & 0.25 & 0.2 & 1,500 & 60 & 235.4 & 0.154 \\
\hline 26 & 0.4 & 0.2 & 1,000 & 60 & 248.2 & 0.176 \\
\hline 27 & 0.25 & 0.35 & 1,000 & 60 & 242.7 & 0.173 \\
\hline 28 & 0.1 & 0.2 & $\mathrm{I}, 500$ & 20 & 281.3 & 0.219 \\
\hline 29 & 0.25 & 0.05 & 1,000 & 60 & 368.7 & 0.33 \\
\hline
\end{tabular}

radiation source. Measurements were taken at $40 \mathrm{kV}$ and $25 \mathrm{~mA}$. The scanning angle was set from $3^{\circ} \leq 2 \theta \leq 40^{\circ}$, and the scanning rate was $2 \% \mathrm{~min}$.

\section{In vitro dissolution studies}

The compendial dissolution test method, Ch.P 2010 Apparatus II, was used with a paddle rotation speed of $75 \mathrm{rpm}$, using $900 \mathrm{~mL}$ of a $\mathrm{HCl}$ solution ( $\mathrm{pH} 1.2$ ), acetate buffer ( $\mathrm{pH} 4.5)$ or PBS (pH 6.8) (all three media containing 0.1\% sodium dodecyl sulfate [SDS] aqueous solution) as the dissolution medium. The dissolution tests for the ADG coarse powder, PM, ADG dripping pills and the freeze-dried ADG-NS were performed using a ZRS-8G dissolution apparatus (Tianjin Tiandatianfa Technology Co., Tianjin, China). Samples equivalent to $\sim 15 \mathrm{mg}$ of ADG were directly added to $900 \mathrm{~mL}$ of a dissolution medium at $37^{\circ} \mathrm{C} \pm 5^{\circ} \mathrm{C}$. At predetermined intervals $(5,10,20$, 30,45 and $60 \mathrm{~min}$ ), $5 \mathrm{~mL}$ of aliquots were withdrawn and the same volume of fresh medium was added to maintain a constant sample volume. Each sample was passed through a $0.10 \mu \mathrm{m}$ cellulose acetate membrane and immediately assayed by high performance liquid chromatography (HPLC). Tests were done on three replicates, and the average and standard deviation (SD) were calculated.

The HPLC method was used for the estimation of ADG in dissolution experiments. The HPLC system comprised a Waters 2695 separation module equipped with a quaternary pump, an autosampling unit and a Waters 2489 UV detector. A Benetnach- $C_{18}(250 \times 4.6 \mathrm{~mm}$ id, $5 \mu \mathrm{m})($ Kromasil ${ }^{\circledR}$; AkzoNobel, Bohus, Sweden) analytical column was used for the estimation. The mobile phase consisted of methanol and water at a ratio of $65: 35(\mathrm{v} / \mathrm{v})$. The flow rate was maintained at $1.0 \mathrm{~mL} / \mathrm{min}$, and the $\mathrm{UV}$ detector was set to $225 \mathrm{~nm}$.

\section{Short-term stability study}

The stability of the freeze-dried ADG-NS was assessed by tightly sealing them in glass containers and keeping them in a drug stability test chamber (Climacell 222; MMM Group, Planegg, Germany) at $40^{\circ} \mathrm{C}$ and relative humidity (RH) $75 \%$ for 1 month. The mean particle size was considered to be an indicator of formulation stability and was measured on the 10th, 20th and 30th days. 


\section{Transport across a Caco-2 cell monolayer}

Caco-2 permeability testing provides guidance on the bioavailability to be expected in vivo. For transport studies, Caco-2 cells were cultured on tissue culture-treated polycarbonate Transwell inserts (Corning, NY, USA) at a density of $1 \times 10^{5}$ cells/well in six-well plates. The cells were allowed to reach confluence and differentiate in the complete medium, forming monolayers 21 days after seeding. The integrity of the Caco-2 cell monolayer was verified by measuring the transepithelial electrical resistance (TEER) with a Millicell ${ }^{\circledR}$ ERS-2 Voltohmmeter (Millipore). TEER values $>350 \Omega \mathrm{cm}^{2}$ were taken as a confirmation of monolayer confluence. Before the experiments, both compartments of the Caco-2 monolayers were preincubated at $37^{\circ} \mathrm{C}$ for $30 \mathrm{~min}$ and then rinsed three times with preheated drug-free transport medium (Hanks' balanced salt solution [HBSS]). The transport solutions containing ADG coarse powder, ADG coarse powder + verapamil, the freeze-dried ADG-NS without TPGS, the freeze-dried ADG-NS without TPGS + verapamil and the freeze-dried ADG-NS were then added to the apical (AP) side, while the receiving compartment contained the corresponding volume of blank transport medium. Samples $(0.4 \mathrm{~mL})$ were collected from the basolateral (BL) compartments at prescheduled time intervals $(0,30,60,90$ and $120 \mathrm{~min})$, and an equal volume of fresh medium was supplied immediately. The electrical resistance was measured before and after the experiments. The apparent permeability coefficient $\left(P_{\text {app }}, \mathrm{cm} / \mathrm{s}\right)$ was calculated using the following equation:

$$
P_{\text {app }}=(\mathrm{dQ} / \mathrm{dt}) / \mathrm{A} \times \mathrm{C}_{0}
$$

where $\mathrm{dQ} / \mathrm{dt}$ is the linear appearance rate of the compound on the receiving side $(\mu \mathrm{g} / \mathrm{s}), \mathrm{C}_{0}$ is the initial concentration in the donor chamber $(\mathrm{ng} / \mathrm{mL})$ and $\mathrm{A}$ is the surface area of the cell monolayers $\left(\mathrm{cm}^{2}\right)$.

\section{Pharmacokinetic study}

Thirty male Sprague Dawley male rats weighing $240 \pm 20 \mathrm{~g}$ were supplied by the Animal Center of Nanjing Medical University (Nanjing, China). They were housed under environmentally controlled conditions at $25^{\circ} \mathrm{C} \pm 2^{\circ} \mathrm{C}$, with $50 \% \pm 10 \%$ relative humidity and a $12 \mathrm{~h}$ light-dark cycle. The rats were permitted free access to food and water until $12 \mathrm{~h}$ prior to experiments. Animal welfare and experimental procedures were performed strictly in accordance with the Guide for the Care and Use of Laboratory Animals and the ethics regulations of Nanjing University of Chinese Medicine.
Rats were randomly divided into five groups, such as ADG coarse powder, PM, ADG dripping pills, the freezedried ADG-NS without TPGS and the freeze-dried ADG-NS, with six animals in each group. Prior to the experiment, the rats were fasted for $12 \mathrm{~h}$ with free access to water. Before the rats were treated by gavage, all the above formulations were dispersed homogeneously in a $0.5 \%$ sodium carboxyl methyl cellulose (CMC-Na) aqueous solution to form suspensions. The same dose of ADG (equivalent to $40 \mathrm{mg}$ of $\mathrm{ADG} / \mathrm{kg}$ ) was given to the rats in these five groups. The blood samples of $\sim 0.3 \mathrm{~mL}$ were collected from the vein of the eyeground $5,10,20,30,45,60,120,240,360,480,600$ and $720 \mathrm{~min}$ after oral administration. The collected blood samples were placed in heparinized tubes and then separated immediately by centrifugation at 3,000 rpm for $10 \mathrm{~min}$. The obtained plasma was transferred into $1.5 \mathrm{~mL}$ Eppendorf tubes and then stored at $-20^{\circ} \mathrm{C}$ until liquid chromatography/tandem mass spectrometry (LC-MS/MS) analysis.

A simple liquid-liquid extraction (LLE) method was applied to extract ADG from rat plasma. First, $10 \mu \mathrm{L}$ of an internal standard (IS) working solution and $10 \mu \mathrm{L}$ of methanol (the volume of the corresponding working solution for the calibration curve and quality control samples) were added to $100 \mu \mathrm{L}$ of rat plasma. The mixture was vortexed for $1 \mathrm{~min}$ and extracted with $1 \mathrm{~mL}$ of ethyl acetate by shaking on a vortex mixer for $10 \mathrm{~min}$ at room temperature. The upper layer was transferred to a clean tube after centrifugation at 4,000 rpm for $5 \mathrm{~min}$. The obtained organic supernatant was evaporated to dryness under a gentle stream of nitrogen. The obtained residue was reconstituted in $100 \mu \mathrm{L}$ of $70 \%$ methanol and centrifuged at $14,000 \mathrm{rpm}$ for $5 \mathrm{~min}$. Subsequently, $5 \mu \mathrm{L}$ of samples were injected into the LC-MS/MS system for analysis.

The concentration of ADG in the plasma was measured by an LC-MS/MS system consisting of an Acquity UPLC system and a Xevo Triple Quadrupole MS (Waters Co., Milford, MA, USA) equipped with an electrospray ionization (ESI) source. The separation was performed on an Acquity UPLC HSS T $_{3} \mathrm{C}_{18}$ column $(100 \times 2.1 \mathrm{~mm}, 1.8 \mu \mathrm{m}$; Waters, Wexford, Ireland) maintained at $40^{\circ} \mathrm{C}$. The mobile phase was composed of methanol and water at a ratio of $70: 30(\mathrm{v} / \mathrm{v})$. The flow rate was $0.35 \mathrm{~mL} / \mathrm{min}$, and the injection volume was $5 \mu \mathrm{L}$. The total run time for LC-MS/MS analysis was $2.5 \mathrm{~min}$.

Noncompartmental pharmacokinetic analysis was used to determine the area under the curve of plasma concentration versus time from zero to the last sampling time $\left(\mathrm{AUC}_{0-t}\right)$ and infinity $\left(\mathrm{AUC}_{0-\infty}\right)$, as well as the elimination half-life $\left(T_{1 / 2}\right)$. Peak drug concentrations $\left(C_{\max }\right)$ and peak times $\left(T_{\max }\right)$ were obtained directly from the plasma drug concentration time profiles. 


\section{In vivo anti-inflammatory studies}

The Carr-induced paw edema model was used to study antiinflammatory activity in mice as previously reported. ${ }^{28}$ All procedures were performed in accordance with the Guide for the Care and Use of Laboratory Animals and the ethics regulations of Nanjing University of Chinese Medicine.

Six groups (10 animals in each group) of male Kunming mice (20-25 g) were treated once daily by gavage with aspirin $(150 \mathrm{mg} / \mathrm{kg}$ ), ADG coarse powder (equivalent to $40 \mathrm{mg}$ of $\mathrm{ADG} / \mathrm{kg}$ ), $\mathrm{ADG}$ dripping pills (equivalent to $40 \mathrm{mg}$ of $\mathrm{ADG} / \mathrm{kg}$ ) and the freeze-dried ADG-NS (equivalent to $40 \mathrm{mg}$ of $\mathrm{ADG} / \mathrm{kg}$ ), which were dispersed homogeneously in $0.5 \%$ CMC-Na to form a suspension. The control and experimental groups were given water once in a day for 4 consecutive days. On the fourth day, $30 \mathrm{~min}$ after the last administration measurement of the initial right hind paw volume, 1\% Carr $(20 \mu \mathrm{L})$ was injected into the plantar side of the right hind paws of the mice, except for the control groups. The paw volume was measured at $0.5,1,2,3,4$ and $5 \mathrm{~h}$ intervals after the Carr injection using a precision micrometer. The rate of paw swelling was calculated according to the following equation:

$$
\text { Paw-swelling rate }(\%)=\frac{a-b}{b} \times 100
$$

where a is the volume of the right hind paw after Carr treatment and $b$ is the volume of the right hind paw before Carr treatment.

After $5 \mathrm{~h}$, the animals were sacrificed and the blood was collected. The collected blood samples were placed in Eppendorf tubes for $2 \mathrm{~h}$, and the cells were then separated by centrifugation at 4,000 rpm for $10 \mathrm{~min}$ to obtain the serum. The serum was taken for the assay kit according to the manufacturer's instructions to investigate the effects on the SOD activity and the levels of NO, IL-1 and TNF- $\alpha$.

\section{Statistical analysis}

Pharmacokinetic data analysis was carried out using the drug and statistics software $\left(\mathrm{DAS}^{\circledR} 2.0\right.$; Boying Corporation, China). Data are expressed as the mean \pm SD. Paired $t$-tests were used to statistically analyze groups using the SPSS 19.0 software (IBM Corp., Armonk, NY, USA). $P$-values $<0.05$ were considered to be a significant difference.

\section{Results and discussion Optimization of ADG-NS}

\section{Experimental design}

The experiment was designed according to the BBD, as shown in Table 2, with 29 runs performed to optimize the four parameters. It was observed that the best-fit model was the quadratic model for each response. The coefficients of the quadratic models and their corresponding $P$-values are shown in Table 3.

For the particle size and PDI responses, the experimental results were fitted to the second-order polynomial equations as follows:

$$
\begin{aligned}
& \text { Particle size }= 223.60+2.23 \mathrm{~A}-119.72 \mathrm{~B} \\
&-6.06 \mathrm{C}-32.44 \mathrm{D}+17.18 \mathrm{AB} \\
&-3.27 \mathrm{AC}-8.35 \mathrm{AD}-10.05 \mathrm{BC} \\
&-13.63 \mathrm{BD}+14.54 \mathrm{CD}+10.09 \mathrm{~A}^{2} \\
&+111.83 \mathrm{~B}^{2}-11.01 \mathrm{C}^{2}+60.86 \mathrm{D}^{2} \\
& \mathrm{PDI}=0.15+3.306 \mathrm{E}-003 \mathrm{~A}-0.073 \mathrm{~B}+7.778 \mathrm{E} \\
&-004 \mathrm{C}-9.000 \mathrm{E}-003 \mathrm{D}-0.027 \mathrm{AB}-1.500 \mathrm{E} \\
&-003 \mathrm{AC}-7.833 \mathrm{E}-003 \mathrm{AD}+0.029 \mathrm{BC} \\
&-0.015 \mathrm{BD}-0.014 \mathrm{CD}+0.029 \mathrm{~A}^{2}+0.066 \mathrm{~B}^{2} \\
&+9.761 \mathrm{E}-003 \mathrm{C}^{2}+0.027 \mathrm{D}^{2}
\end{aligned}
$$

where A, B, C and D are the TPGS concentration, SLS concentration, agitator speed and milling time, respectively. A positive sign represents a synergistic effect, while a nega-

\begin{tabular}{|c|c|c|c|c|}
\hline \multirow[t]{2}{*}{ Factor } & \multicolumn{2}{|c|}{ Particle size } & \multicolumn{2}{|l|}{ PDI } \\
\hline & $F$ value & $P$-value & $F$ value & $P$-value \\
\hline A & 0.03 & 0.8735 & 0.11 & 0.7472 \\
\hline B & 75.58 & $<\mathbf{0 . 0 0 0 I}$ & 53.17 & $<0.0001$ \\
\hline C & 0.19 & 0.6667 & 0.01 & 0.9394 \\
\hline $\mathrm{D}$ & 5.55 & 0.0336 & 0.80 & 0.3858 \\
\hline$A B$ & 0.52 & 0.4831 & 2.45 & 0.1399 \\
\hline$A C$ & 0.02 & 0.8930 & 0.01 & 0.9326 \\
\hline$A D$ & 0.12 & 0.7315 & 0.20 & 0.6597 \\
\hline$B C$ & 0.18 & 0.6799 & 2.73 & 0.1210 \\
\hline $\mathrm{BD}$ & 0.33 & 0.5769 & 0.70 & 0.4164 \\
\hline$C D$ & 0.37 & 0.5519 & 0.67 & 0.4269 \\
\hline$A^{2}$ & 0.02 & 0.8993 & 4.59 & 0.0502 \\
\hline $\mathrm{B}^{2}$ & 35.64 & $<0.0001$ & 23.39 & 0.0003 \\
\hline$C^{2}$ & 0.35 & 0.5661 & 0.51 & $0.487 \mid$ \\
\hline$D^{2}$ & 10.56 & 0.0058 & 3.85 & 0.0698 \\
\hline
\end{tabular}
tive sign indicates an antagonistic effect. In Equation 4, the negative coefficients of $\mathrm{B}, \mathrm{C}$ and $\mathrm{D}$ in the model refer to a decrease in the particle size with an increasing SLS concentration, agitator speed and milling time, respectively. A positive coefficient of $A$ indicates an increase in the particle size with an increasing TPGS concentration. In the case of Equation 5, the negative coefficients of B and D in the model refer to a decrease in PDI with an increasing SLS

Table 3 Coefficients of the quadratic models and their corresponding $P$-values

Note: Values in boldface represent significant terms $(P<0.05)$.

Abbreviation: PDI, polydispersity index. 
concentration and milling time, respectively. The positive coefficients of $\mathrm{A}$ and $\mathrm{C}$ indicate an increase in PDI with an increasing TPGS concentration and agitator speed.

\section{Effect of formulation parameters on particle size and PDI}

Figure $1 \mathrm{~A}$ and $\mathrm{B}$ presents the effects of the TPGS and SLS concentrations on the ADG-NS particle size and PDI. Increasing SLS concentrations caused a gradual decrease in the particle size and PDI. A further increase in the SLS concentration above the optimum level, however, increased the particle size and PDI. This result could be explained by the fact that an optimum amount of stabilizer is required to provide stabilization, but after a point, further addition of the stabilizer probably increases the free concentration of SLS in the system, which might be in the form of an SLS micelle. Therefore, a concentration higher than the critical micelle concentration (CMC) could actually result in less surfactant adsorption, which would further destabilize the nanosuspensions and contribute to an increased particle size and PDI.

A

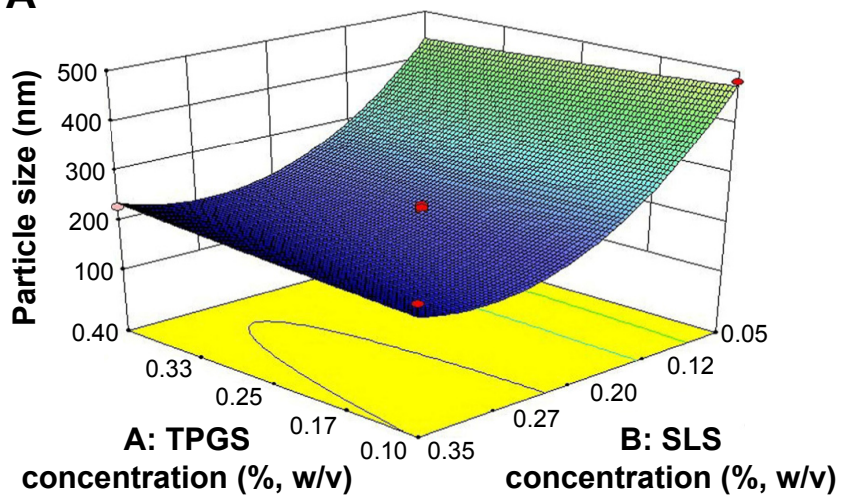

C

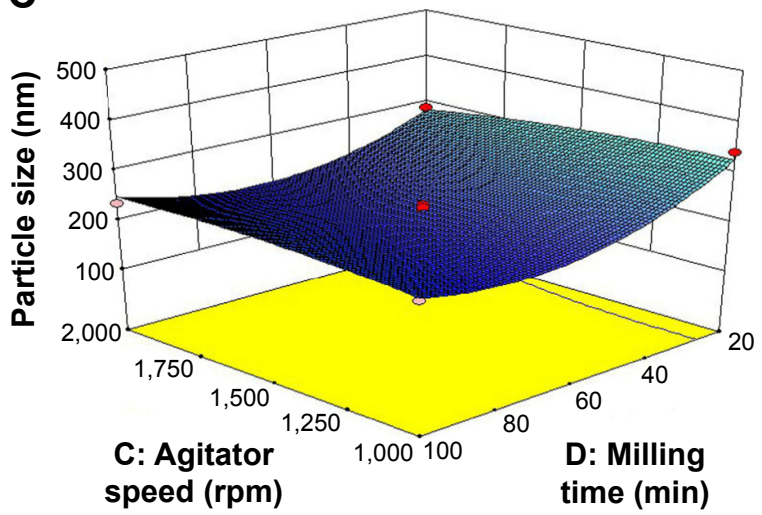

Effect of process parameters on particle size and PDI

As shown in Figure 1C and D, the ADG-NS particle size decreases with an increasing milling time. This relation could be attributed to the increased collision time between the milling media and drug particles, leading to smaller particles. The effect of the agitator speed and milling time on the PDI of ADG-NS is shown in Figure 1D. The results show that the agitator speed and milling time did not have a significant influence on PDI within the scope of the investigation.

\section{Model verification}

The desirability function was evaluated by the Design-Expert software to obtain the optimized ADG-NS formulation. The mean diameter of the drug particle was $215.6 \pm 3.3 \mathrm{~nm}$, and the PDI was $0.165 \pm 0.020$. A low PDI indicates a narrow size distribution, suggesting the presence of a homogenous population within the system, whereas a PDI of $>0.3$ indicates a very broad size distribution. The model verification results are presented in Table 4 with a comparison to the observed

B

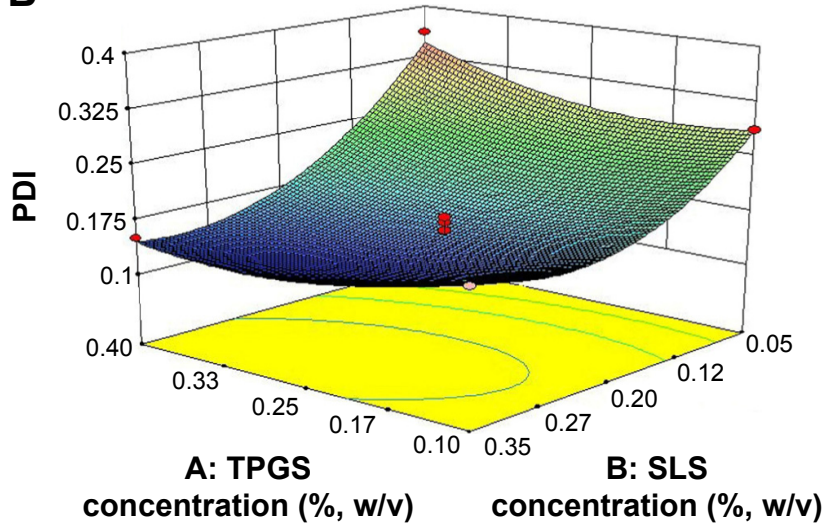

D

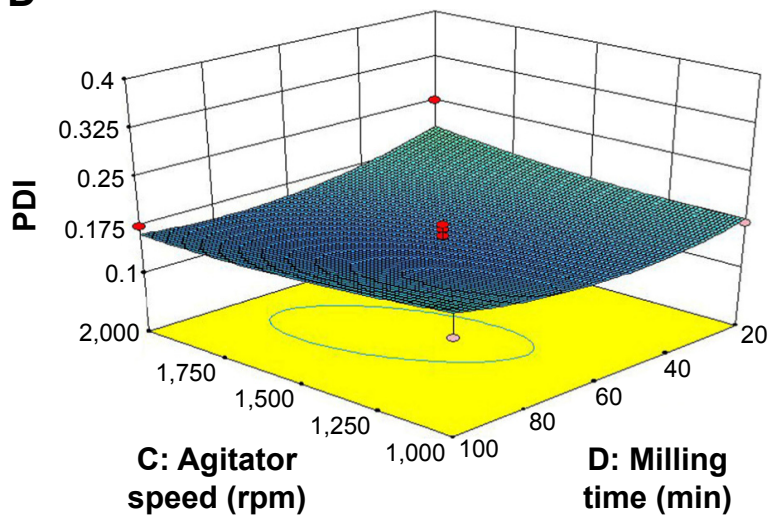

Figure I 3D response surface plots.

Notes: Effect of TPGS concentration and SLS concentration on particle size (A) and PDI (B); effect of agitator speed and milling time on particle size (C) and PDI (D). Abbreviations: 3D, three dimensional; PDI, polydispersity index; SLS, sodium lauryl sulfate; TPGS, D- $\alpha$-tocopheryl polyethylene glycol I000 succinate. 
Table 4 Comparison of predicted and observed values of responses

\begin{tabular}{|c|c|c|c|c|c|c|c|}
\hline \multicolumn{4}{|l|}{ Factors } & \multicolumn{2}{|c|}{ Predicted value } & \multicolumn{2}{|c|}{ Observed value } \\
\hline $\begin{array}{l}\text { TPGS concentration } \\
(\%, w / v)\end{array}$ & $\begin{array}{l}\text { SLS concentration } \\
(\%, w / v)\end{array}$ & $\begin{array}{l}\text { Agitator } \\
\text { speed (rpm) }\end{array}$ & $\begin{array}{l}\text { Milling } \\
\text { time (min) }\end{array}$ & $\begin{array}{l}\text { Particle } \\
\text { size }(\mathrm{nm})\end{array}$ & PDI & $\begin{array}{l}\text { Particle } \\
\text { size }(\mathrm{nm})\end{array}$ & PDI \\
\hline 0.30 & 0.32 & 1,056 & 68 & 214.0 & 0.146 & $215.6 \pm 3.30$ & $0.165 \pm 0.020$ \\
\hline
\end{tabular}

Abbreviations: PDI, polydispersity index; SLS, sodium lauryl sulfate; TPGS, D- $\alpha$-tocopheryl polyethylene glycol 1000 succinate.

values, suggesting that these model equations could be used to predict the ADG-NS particle size and PDI.

\section{Characterization of optimized ADG-NS}

\section{Reconstitution test}

Reconstitution is expressed in terms of the RDI. The mean particle size, PDI and zeta potential are $215.6 \pm 3.3 \mathrm{~nm}$, $0.165 \pm 0.02$ and $-36.9 \pm 2.8 \mathrm{mV}(\mathrm{n}=3)$, respectively, for the liquid ADG-NS prior to freeze drying and $244.6 \pm 3.0 \mathrm{~nm}$, $0.189 \pm 0.016$ and $-37.6 \pm 0.72 \mathrm{mV}(\mathrm{n}=3)$, respectively, for redispersed ADG-NS. The RDI was $113 \% \pm 1.14 \%$, which showed that the freeze-dried ADG-NS powder could be redispersed well.

A

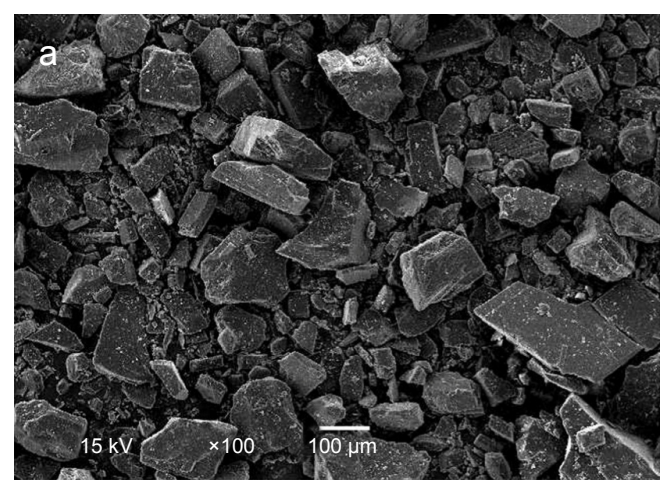

B

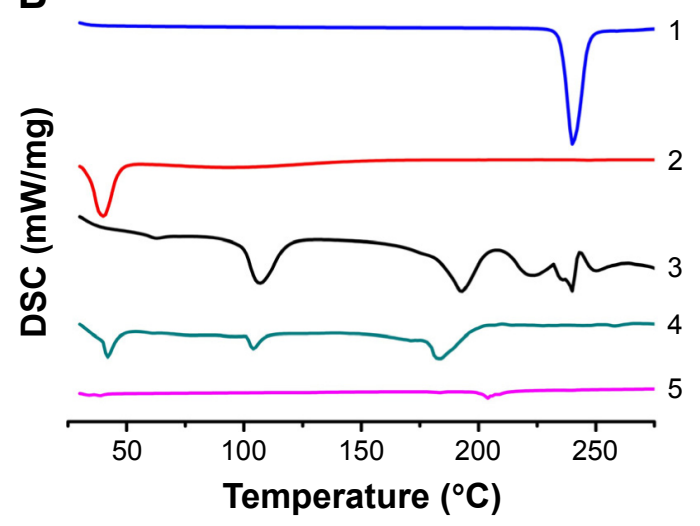

\section{SEM}

To characterize the morphology of the ADG coarse powder and the freeze-dried ADG-NS, SEM imaging was performed. As shown in Figure 2A, there were significant differences in the morphologies between these two samples. The freezedried ADG-NS had a granular and rod-shaped appearance and had no self-adhesion, while the ADG coarse powder had an irregular shape with a great deal of angularities and obvious adhesion. Compared to the ADG coarse powder, whose particle size was $10-200 \mu \mathrm{m}$, the nanosuspensions had a much smaller size $(0.1-0.3 \mu \mathrm{m})$. SEM images indicated that the particles of the freeze-dried ADG-NS were different from those in the ADG coarse powder in terms of not
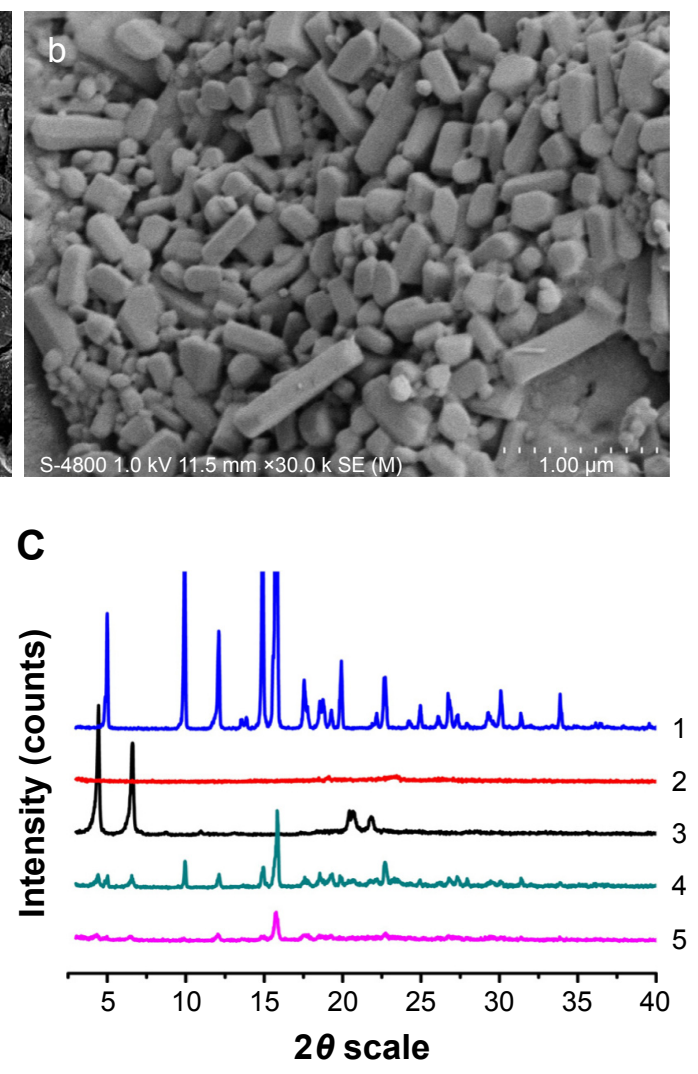

Figure 2 SEM images of the ADG coarse powder (Aa) and the freeze-dried ADG-NS (Ab) and DSC thermograms (B) and PXRD diffractograms (C) of ADG coarse powder (I), TPGS (2), SLS (3), physical mixture of ADG coarse powder, TPGS and SLS (4) and the freeze-dried ADG-NS (5).

Abbreviations: ADG, andrographolide; ADG-NS, ADG nanosuspensions; DSC, differential scanning calorimetry; PXRD, powder X-ray diffraction; SEM, scanning electron microscopy; SLS, sodium lauryl sulfate; TPGS, D- $\alpha$-tocopheryl polyethylene glycol 1000 succinate. 
only size but also shape, which may have influenced their dissolution behavior.

\section{DSC}

DSC was used to analyze the potential changes in the physical state of the freeze-dried ADG-NS due to the high energy input during the process. As shown in Figure 2B, the ADG coarse powder exhibited a single sharp endothermic peak at $240^{\circ} \mathrm{C}$ that was ascribed to the melting point $\left(T_{\mathrm{m}}\right)$ of the drug. SLS exhibited obvious melting processes at 107 and $193^{\circ} \mathrm{C}$, while TPGS had a melting point at $40^{\circ} \mathrm{C}$, which implied a crystalline form for these two substances. However, the endothermic peaks of SLS, TPGS and ADG disappeared in the freeze-dried ADG-NS, which revealed that SLS, TPGS and ADG transform into an amorphous state after the process. Such amorphous excipients may be present on the surface of nanosized ADG, resulting in a reduced crystallinity.

\section{PXRD}

To further confirm the physical state of the components, PXRD was performed to analyze the ADG coarse powder, TPGS, SLS, PM and the freeze-dried ADG-NS. As seen in Figure $2 \mathrm{C}$, ADG coarse powder exhibited sharp, distinctive peaks at the $2 \theta$ angles of $5.00,9.94,12.10,14.90,15.76$, $17.54,19.92,22.72,30.08$ and $33.88^{\circ}$ that were attributed to its crystalline nature. The crystalline nature of ADG in the PM was still maintained, but its diffraction intensities were reduced. This slight change could be due to the added excipients, which can influence the peak intensities through interactions with the drug at specific angles. However, the crystalline nature of ADG was absent from the freeze-dried ADG-NS. As milling is a high-energy process, it tends to cleave the crystal lattice at the weakest sites and reduce the particle size, damaging ADG's crystalline nature. ${ }^{29}$ The intense crystalline peaks at $4.44,6.62,20.22$ and $21.82^{\circ}$ observed in the PXRD spectrum of SLS were not decreased in the spectrum of the freeze-dried ADG-NS, implying a transition to an amorphous phase of SLS.

\section{In vitro dissolution studies}

Figure 3 displays the in vitro dissolution profiles of $\mathrm{ADG}$ coarse powder, $\mathrm{PM}, \mathrm{ADG}$ dripping pills and the freeze-dried ADG-NS in $\mathrm{HCl}$ (pH 1.2) (Figure 3A), acetate buffer ( $\mathrm{pH} 4.5$ ) (Figure 3B) and phosphate buffer ( $\mathrm{pH}$ 6.8) (Figure 3C) (all three media containing $0.1 \%$ SDS).

As seen in Figure 3, within each group, the dissolution in vitro profile of ADG was similar in all three media, and no significant difference was observed. Compared to the ADG coarse powder, the freeze-dried ADG-NS, PM and ADG dripping pills showed a fast dissolution rate. The dissolution of the freeze-dried ADG-NS was $>85 \%$ within the first $10 \mathrm{~min}$, while those of the ADG coarse powder, PM and ADG dripping pills were $<25,65$ and $65 \%$, respectively.

According to the modified Noyes-Whitney equation, ${ }^{30}$ the dissolution rate of a drug is directly proportional to its effective surface area and diffusion coefficient and inversely proportional to the diffusion distance. First, when the particle size of ADG was reduced to the nanoscale, the overall surface area of all the particles increased sharply. Second, an increase in the surface wetting by cogrinding the stabilizer into the suspension could also result in a further increase in the dissolution rate. The hydrophilic surfactant (TPGS and SLS) that was used created a hydrophilic environment around the ADG microcrystals, effectively wetted the drug and then increased the diffusion coefficient. Third, the diffusion distance decreases with a reduction in the particle size according
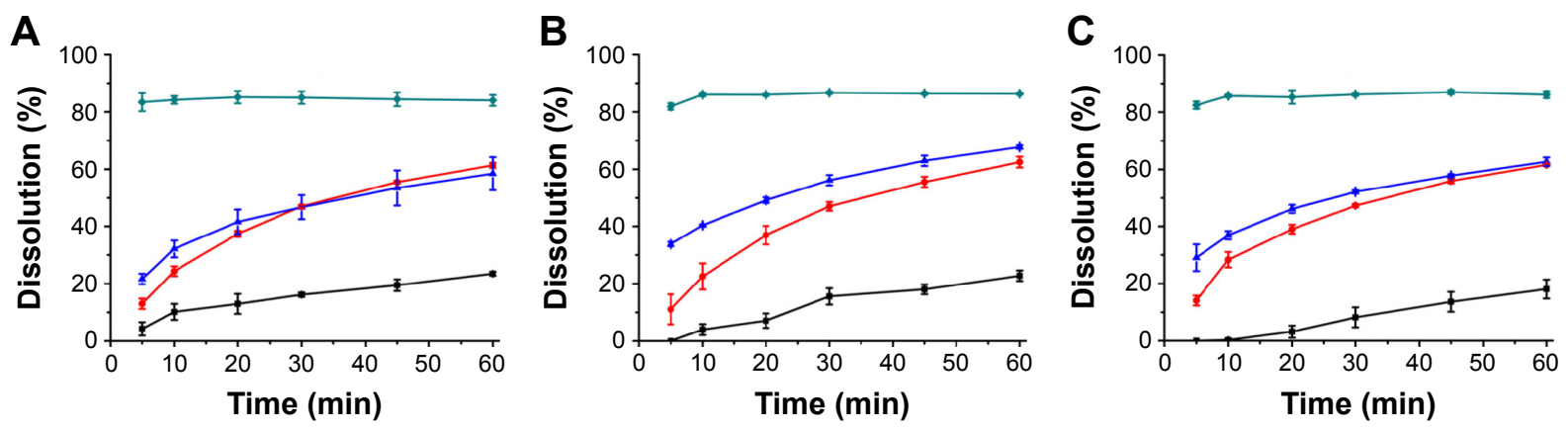

$\rightarrow$ ADG coarse powder $\leftarrow$ PM $\rightarrow$ ADG dripping pills $\rightarrow$ Freeze-dried ADG-NS

Figure 3 In vitro dissolution profiles of ADG coarse powder, PM, ADG dripping pills and the freeze-dried ADG-NS in HCl (pH I.2) (A), acetate buffer (pH 4.5) (B) and phosphate buffer ( $\mathrm{pH}$ 6.8) (C) (all three media containing 0.1\% SDS) ( $n=3$, mean $\pm \mathrm{SD})$.

Abbreviations: ADG, andrographolide; ADG-NS, ADG nanosuspensions; $\mathrm{HCl}$, hydrochloric acid; PM, physical mixture of the ADG coarse powder, TPGS and SLS; SD, standard deviation; SDS, sodium dodecyl sulfate; SLS, sodium lauryl sulfate; TPGS, d- $\alpha$-tocopheryl polyethylene glycol 1000 succinate. 
to the Prandtl equation. ${ }^{31,32}$ Finally, an improvement in the saturation solubility due to the amorphous structure of ADG also contributes to an increased dissolution rate. ${ }^{33,34}$

\section{Short-term stability study}

Changes in the particle size and PDI of the ADG-NS were investigated to determine whether the freeze-dried ADG-NS had short-term stability. The results showed no significant changes in the mean particle size or PDI over 1 month, which indicated that nanosized ADG could remain highly stable in nanosuspensions.

\section{Transport across a Caco-2 cell monolayer}

A good correlation between the oral absorption of a drug in human and its apparent permeability coefficient through Caco- 2 cells has been calculated and described by several researchers since its first report in $1990 .^{35}$

As presented in Table 5, it is obvious that the presence of verapamil could significantly increase the permeation of ADG in the ADG coarse powder + verapamil and the freezedried ADG-NS without TPGS + verapamil groups $(P<0.01)$. These results suggest that ADG may be a substrate of P-gp, which is consistent with previous reports. ${ }^{11}$

Compared to the ADG coarse powder group, the freezedried ADG-NS (with or without TPGS) showed a significant increase in the membrane permeability $\left(P_{\text {app }}\right)(P<0.01)$. The enhanced dissolution rate and an increased solubility of the nanosuspensions may modify the permeability of ADG through intestinal epithelial cells. ${ }^{36}$ One of the notable characteristics of nanosuspensions is their potential to increase the dissolution rate, which may alter the cell membrane permeation behavior in vitro and consequently affect the absorption in vivo. ${ }^{32}$

In addition, the freeze-dried ADG-NS (with TPGS) exhibited significantly higher permeability across cell

Table 5 Apparent permeability coefficients $\left(P_{\text {app }}\right)$ of ADG coarse powder and nanosuspensions through a Caco-2 cell monolayer during AP-BL transport $(n=3$, mean $\pm S D)$

\begin{tabular}{ll}
\hline Groups & $\boldsymbol{P}_{\text {app }} \times 10^{-6}(\mathbf{c m} / \mathbf{s})$ \\
\hline ADG coarse powder & $1.69 \pm 0.16$ \\
ADG coarse powder + verapamil & $2.54 \pm 0.18^{* *}$ \\
Freeze-dried ADG-NS without TPGS & $5.1 \pm 0.55^{* *}$ \\
Freeze-dried ADG-NS without TPGS + verapamil & $9.08 \pm 1.11^{* *} \ldots$ \\
Freeze-dried ADG-NS & $9.54 \pm 0.61^{* *}$ \\
\hline
\end{tabular}

Notes: $* * P<0.01$ compared to ADG coarse powder. ${ }^{\#} P<0.01$ compared to the freeze-dried ADG-NS without TPGS.

Abbreviations: ADG, andrographolide; AP, apical; BL, basolateral; ADG-NS, ADG nanosuspensions; SD, standard deviation; TPGS, D- $\alpha$-tocopheryl polyethylene glycol 1000 succinate. membranes, with significant enhancements in $P_{\text {app }}(87.1 \%$ higher) compared to the freeze-dried ADG-NS without TPGS, indicating that TPGS, a P-gp inhibitor, decreased drug efflux by P-gp.

\section{Pharmacokinetic study}

The curves of ADG plasma concentration versus time in rats after the oral administration of ADG coarse powder, PM, ADG dripping pills, the freeze-dried ADG-NS without TPGS and the freeze-dried ADG-NS are shown in Figure 4. The corresponding pharmacokinetic parameters are listed in Table 6.

Compared to the ADG coarse powder, the ADG dripping pills, the freeze-dried ADG-NS without TPGS and the freeze-dried ADG-NS exhibited significantly higher exposure in the plasma, with significant enhancements in $C_{\max }(116.3,157.9$ and $203.5 \%$ higher, respectively) and $\mathrm{AUC}_{0-t}(90.98,164.48$ and $308.3 \%$ higher, respectively), which indicated that the dripping pills and nanosuspensions (with or without TPGS) were more easily absorbed in vivo than ADG coarse powder.

In addition, the freeze-dried ADG-NS exhibited significantly higher plasma exposure, with significant enhancements in $\mathrm{AUC}_{0-t}(54.3 \%$ higher), compared to the freeze-dried ADG-NS without TPGS. This result was mainly ascribed to the inhibitory effects of TPGS on the P-gp efflux function. Moreover, the $\mathrm{AUC}_{0-t}$ of the freeze-dried ADG-NS (with or without TPGS) was 1.38 or 2.14 times higher than that of the ADG dripping pills.

An increase in the absorption of the freeze-dried ADG-NS can be explained by the Ostwald-Freundlich and NoyesWhitney equations. The marked reduction in particle size and the high surface area cause more rapid dissolution, thus leading to excellent bioavailability. Moreover, the particle size can influence the speed of particle diffusion through the mucous layer to reach intestinal epithelial cells. ${ }^{37,38}$ In addition, the nanosuspensions have inherent advantages in enhancing oral absorption. First, nanoparticles exhibit high bioadhesion to the intestinal wall that can prolong the retention time in the gastrointestinal tract and increase passive absorption. Second, the uptake of intact gastrointestinal polymeric particles occurs by mechanisms involving M-cells in Peyer's patches of the lymphoid tissues and mesentery nodes. ${ }^{39-41}$

\section{In vivo anti-inflammatory studies Carrageenan-induced paw edema in mice}

In this study, Carr-induced paw edema was used because this model is widely used to evaluate the effects of 


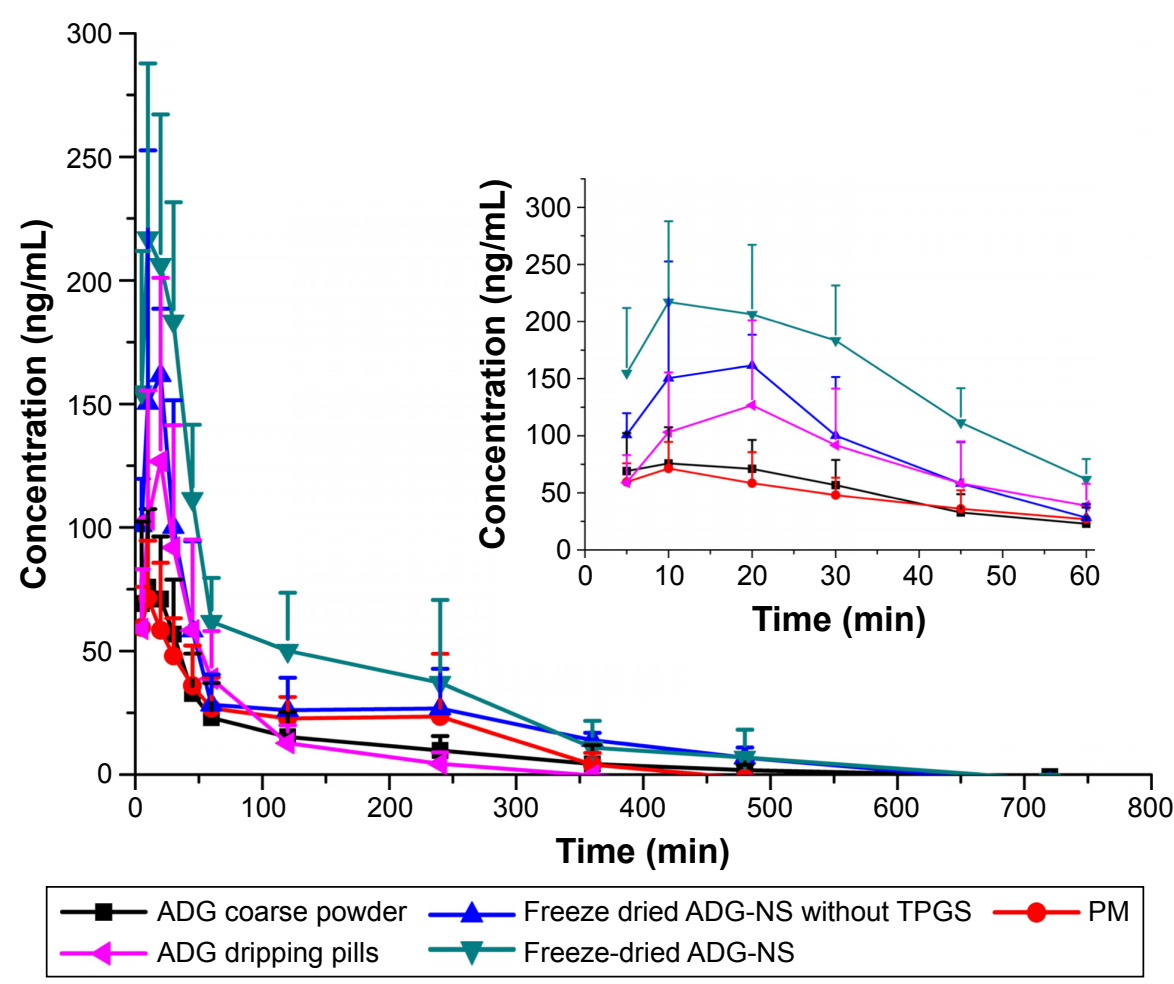

Figure 4 The profiles of mean plasma concentration-time of ADG in rats after oral administration of ADG coarse powder, PM, ADG dripping pills, the freeze-dried ADG-NS without TPGS and the freeze-dried ADG-NS at a dose of $40 \mathrm{mg} / \mathrm{kg}(\mathrm{n}=6$, mean $\pm S D)$.

Abbreviations: ADG, andrographolide; ADG-NS, ADG nanosuspensions; PM, physical mixture of the ADG coarse powder, TPGS and SLS; SD, standard deviation; TPGS, $D$ - $\alpha$-tocopheryl polyethylene glycol 1000 succinate; SLS, sodium lauryl sulfate.

anti-inflammatory drugs. The effects on the paw-swelling rate in Carr-induced mice in different groups at different points are illustrated in Figure 5.

As shown in Figure 5, compared to ADG coarse powder, the freeze-dried ADG-NS was more effective in reducing the paw-swelling rate throughout the whole course of the experiment, from 0.5 to $5 \mathrm{~h}$ after Carr injection $(P<0.01)$. Its maximum effect occurred at $0.5 \mathrm{~h}$, and the rate of paw swelling decreased to $52.45 \%$ of that observed in the control group.

In addition, in comparison to the ADG coarse powder, ADG dripping pills also had a greater effect, but these effects were not significant at all time points. Pretreatment with ADG dripping pills was less effective at reducing the paw-swelling rate than an equivalent dose of the freeze-dried ADG-NS.

\section{SOD activity and NO, IL-I and TNF- $\alpha$ levels in serum}

The effects of the six groups on the SOD activity and the levels of NO, IL- 1 and TNF- $\alpha$ in serum are shown in Figure 6.

As seen in Figure 6, the NO, IL-1 and TNF- $\alpha$ levels increased significantly, while the SOD activity decreased significantly in Carr-induced paw edema at the fifth hour after Carr injection $(P<0.01$ and $<0.05)$ in the control groups.

Table 6 Pharmacokinetic parameters of ADG in rats after oral administration of ADG coarse powder, PM, ADG dripping pills, the freeze-dried ADG-NS without TPGS and the freeze-dried ADG-NS at a dose of $40 \mathrm{mg} / \mathrm{kg}(\mathrm{n}=6$, mean \pm SD)

\begin{tabular}{|c|c|c|c|c|c|}
\hline Parameters & $\begin{array}{l}\text { ADG coarse } \\
\text { powder }\end{array}$ & PM & $\begin{array}{l}\text { ADG dripping } \\
\text { pills }\end{array}$ & $\begin{array}{l}\text { Freeze-dried ADG-NS } \\
\text { without TPGS }\end{array}$ & $\begin{array}{l}\text { Freeze-dried } \\
\text { ADG-NS }\end{array}$ \\
\hline$C_{\max }(\mu g / L)$ & $77.74 \pm 22.17$ & $80.25 \pm 17.35$ & $168.17 \pm 47.28 * *$ & $200.49 \pm 18.91 * *$ & $235.91 \pm 53.73 * *$ \\
\hline$T_{\max }(\min )$ & $28.75 \pm 12.50$ & $22.00 \pm 9.48$ & $22.50 \pm 5.57$ & $20.00 \pm 10.00$ & $12.50 \pm 8.80$ \\
\hline $\mathrm{AUC}_{0-t}(\mu \mathrm{g} / \mathrm{L} \min )$ & $5,379.28 \pm I, 452.5 \mid$ & $9,117.87 \pm 2,126.20^{*}$ & $10,273.45 \pm 1,699.48^{* *}$ & $14,227.02 \pm 306.2447^{* *}$ & $21,960.14 \pm 5,392.04 * * \ldots$ \\
\hline $\mathrm{AUC}_{0-\infty}(\mu \mathrm{g} / \mathrm{L} \mathrm{min})$ & $5,665.4 I \pm I, 654.43$ & $9,|20.34 \pm 2| 3 \mid, .25^{* *}$ & $10,338.11 \pm 1,697.06 * *$ & $15,191.70 \pm 1,640.77^{* *}$ & $22,069.20 \pm 5,484.57^{* *, \#}$ \\
\hline$t_{1 / 2}(\min )$ & $30.02 \pm 8.99$ & $55.83 \pm 12.24 *$ & $101.42 \pm 23.37 * *$ & $165.21 \pm 33.14 * *$ & $90.52 \pm 24.37 * *, \#$ \\
\hline
\end{tabular}

Notes: $* P<0.05$ and $* * P<0.01$ compared to ADG coarse powder. ${ }^{*} P<0.05$ and ${ }^{\# P}<0.01$ compared to the freeze-dried ADG-NS without TPGS.

Abbreviations: ADG, andrographolide; ADG-NS, ADG nanosuspensions; AUC, area under the curve; PM, physical mixture of the ADG coarse powder, TPGS and SLS; SD, standard deviation; TPGS, D- $\alpha$-tocopheryl polyethylene glycol 1000 succinate; SLS, sodium lauryl sulfate. 


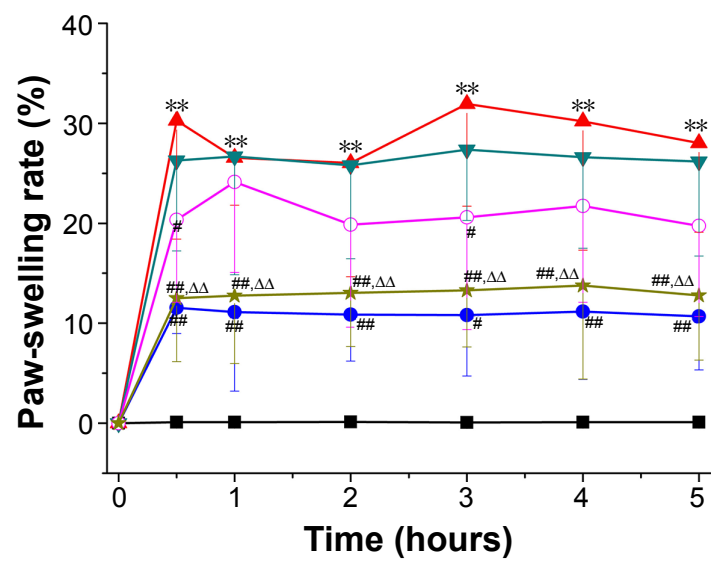

\begin{tabular}{|c|c|}
\hline $\begin{array}{l}- \text { - Control } \\
- \text { Model }\end{array}$ & $\begin{array}{cc}- & -0-\text { Asprin } \\
- \text { ADG coarse }-\star & \text { Areeze-dried } \\
\text { powder } & \text { ADG-NS }\end{array}$ \\
\hline
\end{tabular}

Figure 5 Effects on paw-swelling rate in Carr-induced mice in different groups at different points $(n=10$, mean $\pm S D)$.

Notes: $* * P<0.01$ compared to control. ${ }^{*} P<0.05$ and $\# P<0.01$ compared to model. $\triangle \Delta P<0.0$ l compared to ADG.

Abbreviations: ADG, andrographolide; ADG-NS, ADG nanosuspensions; SD, standard deviation.
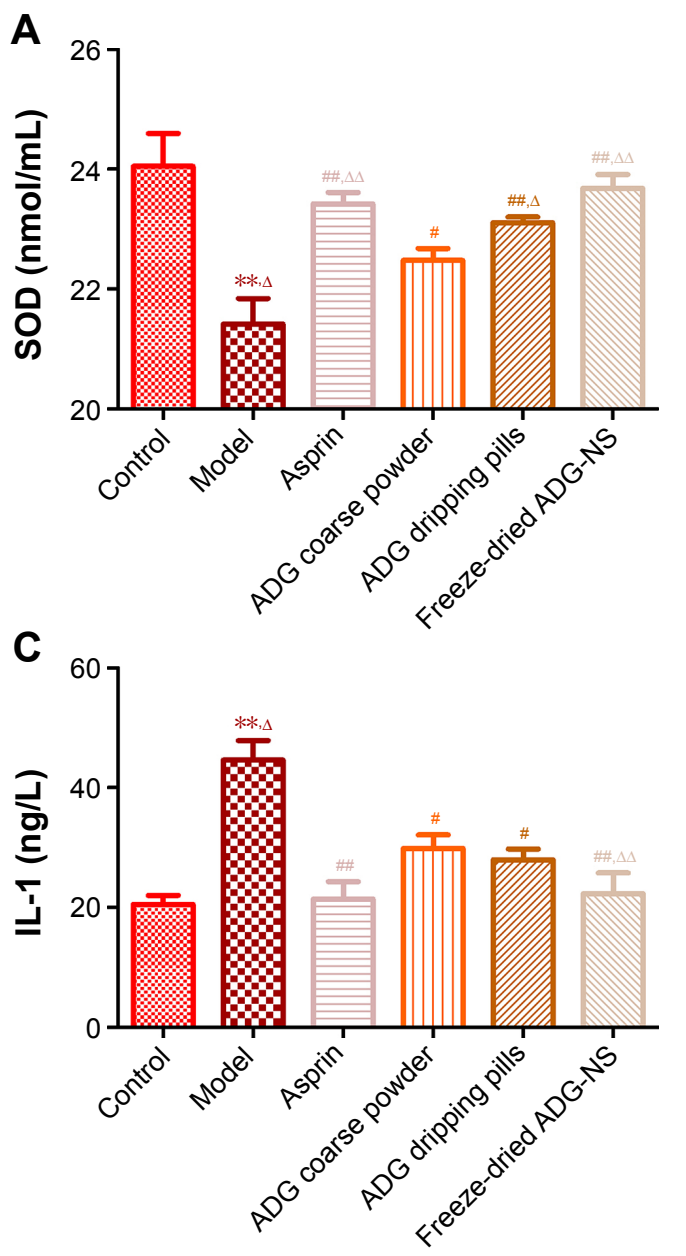

Compared to the control group, a significant reduction in the NO, IL- 1 and TNF- $\alpha$ levels and an increase in the SOD activity were observed in the serum of mice treated with the freeze-dried ADG-NS and ADG dripping pills $(P<0.01$ and $<0.05)$, which indicated that ADG-NS and ADG dripping pills had anti-inflammatory effects.

In comparison to the ADG coarse powder, the freezedried ADG-NS and ADG dripping pills caused a significant decrease in the levels of NO, IL- 1 and TNF- $\alpha$ and an increase in the SOD activity $(P<0.01$ and $<0.05)$. However, compared to the ADG dripping pills, the freeze-dried ADG-NS exhibited better anti-inflammatory potency, with a significant increase in the SOD activity and a decrease in the NO, IL- 1 and TNF- $\alpha$ levels, indicating that the freeze-dried ADG-NS had greater anti-inflammatory potency than the ADG dripping pills.

\section{Conclusion}

In this study, novel amorphous ADG-NS stabilized by TPGS/ SLS were prepared for the first time by a wet media milling
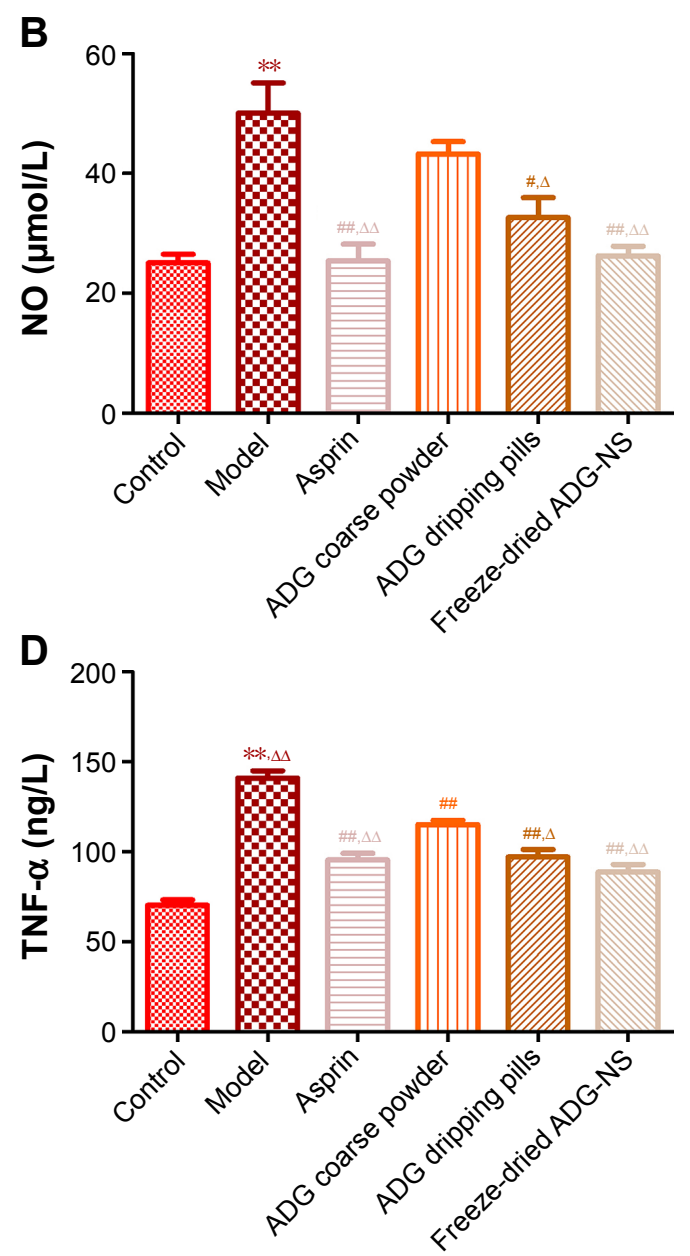

Figure 6 SOD activity (A) and NO (B), IL-I (C) and TNF- $\alpha$ (D) levels in serum in different groups ( $n=10$, mean \pm SD).

Notes: $* * P<0.01$ compared to control. ${ }^{\# P<0.05}$ and ${ }^{\# P} P<0.01$ compared to model. $\triangle P<0.05$ and $\triangle 4 P<0.01$ compared to $A D G$.

Abbreviations: ADG, andrographolide; ADG-NS, ADG nanosuspensions; SOD, superoxide dismutase; NO, nitric oxide; IL-I, Interleukin-I; TNF- $\alpha$, tumor necrosis factor- $\alpha$; SD, standard deviation. 
technique using a response surface methodology design. The freeze-dried ADG-NS dissolved in vitro much faster than the ADG coarse powder and exhibited good redispersibility and high stability. Compared to the ADG coarse powder, ADG-NS exhibited significantly higher membrane permeability $\left(P_{\text {app }}\right)$ across a Caco-2 cell monolayer as well as higher $C_{\max }$ and $\mathrm{AUC}_{0-t}$. Moreover, ADG-NS have a better anti-inflammatory effect on Carr-induced paw edema. This study indicated that nanosuspensions can act as effective delivery devices for ADG to enhance its oral bioavailability and biological efficacy.

\section{Acknowledgments}

The authors acknowledge financial support from the National Natural Science Foundation of China (81503259), the Jiangsu Natural Science Foundation of China (BK20141465 and BK20151002), the Project Funded by the Priority Academic Program Development of Jiangsu Higher Education Institutions and Top-notch Academic Programs Project of Jiangsu Higher Education Institutions (PPZY2015A070).

\section{Disclosure}

The authors report no conflicts of interest in this work.

\section{References}

1. Shi CY, Dong LY. A study on antiinflammation, antipyretic and analgesic action of andrograpolide sodiumbisulphite. Chin J Ethnomed Ethnopharmacy. 2009;18(12):5-6.

2. Yang D, Zhang W, Song L, Guo F. Andrographolide protects against cigarette smoke-induced lung inflammation through activation of heme oxygenase-1. J Biochem Mol Toxicol. 2013;27(5):259-265.

3. Yan YY, Shi GX, Shao J, Wang TM, Wang CZ. Advance in studies on antiinfection of andrographolide and its derivatives in past 10 years. Chin J Chin Materia Med. 2013;38(22):3819-3824.

4. Zhou B, Zhang D, Wu X. Biological activities and corresponding SARs of andrographolide and its derivatives. Mini Rev Med Chem. 2013; 13(2):298-309.

5. Lee YC, Lin HH, Hsu CH, Wang CJ, Chiang TA, Chen JH. Inhibitory effects of andrographolide on migration and invasion in human nonsmall cell lung cancer A549 cells via down-regulation of PI3K/Akt signaling pathway. Eur J Pharmacol. 2010;632(1-3):23-32.

6. Hocker HJ, Cho KJ, Chen CY, et al. Andrographolide derivatives inhibit guanine nucleotide exchange and abrogate oncogenic Ras function. Proc Natl Acad Sci U S A. 2013;110(25):10201-10206.

7. Chen M, Xie C, Liu L. Solubility of andrographolide in various solvents from (288.2 to 323.2) K. J Chem Eng Data. 2010;55(11):5297-5298.

8. Gu Y, Ma J, Liu Y, Chen B, Yao S. Determination of andrographolide in human plasma by high-performance liquid chromatography/mass spectrometry. J Chromatogr B Analyt Technol Biomed Life Sci. 2007; 854(1-2):328-331.

9. Ye L, Wang T, Tang L, et al. Poor oral bioavailability of a promising anticancer agent andrographolide is due to extensive metabolism and efflux by P-glycoprotein. J Pharm Sci. 2011;100(11):5007-5017.

10. He XD, Gong P, Qi CL, He W, Wang LJ, Li WD. The antitumor effects of andrographolide drop pills on murine B16 melanoma. Acad J Guangdong Coll Pharm. 2011;27(2):163-165.

11. Bothiraja C, Shinde MB, Rajalakshmi S, Pawar AP. Evaluation of molecular pharmaceutical and in-vivo properties of spray-dried isolated andrographolide-PVP. J Pharm Pharmacol. 2009;61(11):1465-1472.
12. Ren K, Zhang Z, Li Y, et al. Physicochemical characteristics and oral bioavailability of andrographolide complexed with hydroxypropylbeta-cyclodextrin. Pharmazie. 2009;64(8):515-520.

13. Yang T, Sheng HH, Feng NP, Wei H, Wang ZT, Wang CH. Preparation of andrographolide-loaded solid lipid nanoparticles and their in vitro and in vivo evaluations: characteristics, release, absorption, transports, pharmacokinetics, and antihyperlipidemic activity. J Pharm Sci. 2013; 102(12):4414-4425.

14. Zhang Y, Hu X, Liu XL, et al. Dry state microcrystals stabilized by an HPMC film to improve the bioavailability of andrographolide. Int J Pharm. 2015;493(1-2):214-223.

15. Merisko-Liversidge E, Liversidge GG, Cooper ER. Nanosizing: a formulation approach for poorly-water-soluble compounds. Eur J Pharm Sci. 2003;18(2):113-120.

16. Jacobs C, Müller RH. Production and characterization of a budesonide nanosuspension for pulmonary administration. Pharm Res. 2002;19(2): 189-194.

17. Ponchel G, Montisci MJ, Dembri A, Durrer C, Duchêne D. Mucoadhesion of colloidal particulate systems in the gastro-intestinal tract. Eur $J$ Pharm Biopharm. 1997;44(1):25-31.

18. Kim SC, Kim DW, Shim YH, et al. In vivo evaluation of polymeric micellar paclitaxel formulation: toxicity and efficacy. J Control Release. 2001;72(1-3):191-202.

19. George M, Ghosh I. Identifying the correlation between drug/stabilizer properties and critical quality attributes (CQAs) of nanosuspension formulation prepared by wet media milling technology. Eur J Pharm Sci. 2013;48(1-2):142-152.

20. Shegokar R, Muller RH. Nanocrystals: industrially feasible multifunctional formulation technology for poorly soluble actives. Int J Pharm. 2010;399(1-2):129-139.

21. Van Eerdenbrugh B, Vermant J, Martens JA, et al. A screening study of surface stabilization during the production of drug nanocrystals. J Pharm Sci. 2009;98(6):2091-2103.

22. Guo Y, Luo J, Tan S, Otieno BO, Zhang Z. The applications of vitamin E TPGS in drug delivery. Eur J Pharm Sci. 2013;49(2):175-186.

23. Rege BD, Kao JP, Polli JE. Effects of nonionic surfactants on membrane transporters in Caco-2 cell monolayers. Eur J Pharm Sci. 2002; 16(4-5):237-246.

24. Yu LX. Pharmaceutical quality by design: product and process development, understanding, and control. Pharm Res. 2008;25(4):781-791.

25. Vogt N. Quality by design: managing research and development. Chemometr Intell Lab Syst. 1992;14(1-3):93-101.

26. Kumar S, Gokhale R, Burgess DJ. Quality by design approach to spray drying processing of crystalline nanosuspensions. Int $J$ Pharm. 2014;464(1-2):234-242.

27. Yue PF, Wan J, Wang Y, et al. d-Alpha-tocopherol acid polyethylene glycol 1000 succinate, an effective stabilizer during solidification transformation of baicalin nanosuspensions. Int J Pharm. 2013;443(1-2): 279-287.

28. Deng JS, Huang SS, Lin TH, et al. Analgesic and anti-inflammatory bioactivities of eburicoic acid and dehydroeburicoic acid isolated from Antrodia camphorata on the inflammatory mediator expression in mice. J Agric Food Chem. 2013;61(21):5064-5071.

29. Müller RH, Jacobs C, Kayser O. Nanosuspensions as particulate drug formulations in therapy rationale for development and what we can expect for the future. Adv Drug Deliv Rev. 2001;47(1):3-19.

30. Bikiaris DN. Solid dispersions, part I: recent evolutions and future opportunities in manufacturing methods for dissolution rate enhancement of poorly water-soluble drugs. Expert Opin Drug Deliv. 2011;8(11): $1501-1519$.

31. Pu X, Sun J, Wang Y, et al. Development of a chemically stable 10-hydroxycamptothec in nanosuspensions. Int J Pharm. 2009;379: $167-173$.

32. Mauludin R, Müller RH, Keck CM. Kinetic solubility and dissolution velocity of rutin nanocrystals. Eur J Pharm Sci. 2009;36(4-5):502-510.

33. Dai WG, Dong LC, Song YQ. Nanosizing of a drug/carrageenan complex to increase solubility and dissolution rate. Int J Pharm. 2007; 342(1-2):201-207. 
34. Kumar S, Shen J, Burgess DJ. Nano-amorphous spray dried powder to improve oral bioavailability of itraconazole. J Control Release. 2014; 192:95-102.

35. Artursson P. Epithelial transport of drugs in cell culture. I: a model for studying the passive diffusion of drugs over intestinal absorptive (Caco-2) cells. J Pharm Sci. 1990;79(6):476-482.

36. Wang $\mathrm{Y}$, Zhang D, Liu Z, et al. In vitro and in vivo evaluation of silybin nanosuspensions for oral and intravenous delivery. Nanotechnology.2010; 21(15):155104.

37. Behrens I, Pena AIV, Alonso MJ, Kissel T. Comparative uptake studies of bioadhesive and non-bioadhesive nanoparticles in human intestinal cell lines and rats: the effect of mucus on particle adsorption and transport. Pharm Res. 2002;19(8):1185-1193.
38. Hanafy A, Spahn-Langguth H, Vergnault G, et al. Pharmacokinetic evaluation of oral fenofibrate nanosuspensions and SLN in comparison to conventional suspensions of micronized drug. Adv Drug Deliv Rev. 2007;59(6):419-426.

39. Clark M, Jepson MA, Hirst BH. Exploiting M cells for drug and vaccine delivery. Adv Drug Deliv Rev. 2001;50(1-2):81-106.

40. Ensign LM, Cone R, Hanes J. Oral drug delivery with polymeric nanoparticles: the gastrointestinal mucus barriers. Adv Drug Deliv Rev. 2012;64(6):557-570.

41. Florence AT, Hussain N. Transcytosis of nanoparticle and dendrimer delivery systems: evolving vistas. Adv Drug Deliv Rev. 2001; 50(suppl 1):S69-S89.
International Journal of Nanomedicine

\section{Publish your work in this journal}

The International Journal of Nanomedicine is an international, peerreviewed journal focusing on the application of nanotechnology in diagnostics, therapeutics, and drug delivery systems throughout the biomedical field. This journal is indexed on PubMed Central, MedLine, CAS, SciSearch ${ }^{\circledR}$, Current Contents ${ }^{\circledR} /$ Clinical Medicine,

\section{Dovepress}

Journal Citation Reports/Science Edition, EMBase, Scopus and the Elsevier Bibliographic databases. The manuscript management system is completely online and includes a very quick and fair peer-review system, which is all easy to use. Visit http://www.dovepress.com/ testimonials.php to read real quotes from published authors. 